\title{
Evaluation of selected parameters of oxidative stress in patients with androgenetic alopecia
}

\author{
Anna Cwynar ${ }^{1}$, Dorota Olszewska-Słonina ${ }^{1}$, Rafał Czajkowski ${ }^{2}$
}

${ }^{1}$ Department of Pathobiochemistry and Clinical Chemistry, Collegium Medicum in Bydgoszcz, Nicolaus Copernicus University in Torun, Poland

${ }^{2}$ Department of Dermatology, Sexually Transmitted Disorders and Immunodermatology, Collegium Medicum in Bydgoszcz, Nicolaus Copernicus University in Torun, Poland

Adv Dermatol Allergol 2021; XXXVIII (3): 528-529

DOI: https://doi.org/10.5114/ada.2021.107941

Androgenetic alopecia (AGA) is a type of non-pubescent baldness with progressive thinning of the head hair according to a characteristic pattern [1]. The pathogenesis of androgenetic alopecia includes both genetic and hormonal factors [2].

Some studies suggest that inflammation is the concomitant feature of AGA, although its importance in the pathogenesis of this disease is quite controversial [2, 3]. Activated $T$ cells accumulating within the hair follicles have been observed in head skin biopsies in patients with AGA [4]. At the same time, moderate lymphocytes infiltration occurred in about $40 \%$ of cases of androgenetic alopecia and it was also present in $10 \%$ of healthy controls [5]. Occasionally, the presence of eosinophilia and mast cells could be observed [6]. Inflammation and oxidative stress are closely related in biological systems [2]. Oxidative stress in addition comes to accelerate the aging process as AGA deteriorates with age [7].

To date, Prie et al. have presented one of the few reports indicating a significantly higher plasma malondialdehyde (MDA) concentration in patients with AGA in relation to the control group [2]. The present state may be explained by the inconsistencies in the scientific community regarding the recognition of inflammation as a concomitant feature of AGA [2, 3]. Although histological studies have confirmed the presence of inflammation in the hair follicles, clinically AGA is still considered a noninflammatory disease $[8,9]$.

Current medical literature provides little information on the oxidative stress parameters in the blood of patients with androgenic alopecia, and therefore it was considered interesting to study the antioxidant systems in erythrocytes and plasma of these patients.
This study aims to investigate the role of oxidative stress in cases of AGA and in healthy controls by measuring the levels of plasma and erythrocyte (MDA) and the level of the ferric reducing ability of plasma (FRAP). The study included $21 \mathrm{AGA}$ patients (12 females/9 males, mean age: $45.8 \pm 12$ ) and the control group consisting of 40 ageand sex-matched healthy volunteers ( 25 females/15 males, mean age: $33.9 \pm 11)$. There was no significant body mass index (BMI) difference between the groups.

Malondialdehyde concentration in blood plasma was higher in patients with AGA compared with controls ( $4.55 \%$ and $14.29 \%$, respectively), but these differences were not statistically significant $(p=0.572 ; p=0.132)$. However, significant differences were observed in FRAP levels ( $p=0.028$ ) between the studied group and the healthy volunteers. There was a positive correlation between plasma MDA levels and FRAP in patients with AGA $\left(r_{s}=0.457 ; p=0.037\right)$ (Table 1$)$.

Oxidative stress is a result of inadequate antioxidant defence or overproduction of reactive oxygen species (ROS). Its presence has been demonstrated in many dermatological diseases, including alopecia areata, psoriasis, vitiligo, atopic dermatitis, lichen planus, acne vulgaris, seborrheic dermatitis and skin cancer [10] as well as in androgenetic alopecia [2].

Lipid peroxidation is one of the most important biological processes associated with the action of ROS. Erythrocytes are particularly exposed to oxidative stress [11]. Increased levels of MDA in plasma and erythrocytes in patients with androgenetic alopecia suggest the occurrence of increased oxidative stress and may be associated with the presence of inflammation.

The FRAP evaluation allows the direct determination of the antioxidant capacity of cells and tissues as well as

Address for correspondence: Anna Cwynar PhD, Department of Pathobiochemistry and Clinical Chemistry, Collegium Medicum, Nicolaus Copernicus University, Bydgoszcz, Poland, phone: +48 793414 790, e-mail: anna.cwynar@vp.pl Received: 5.03.2020, accepted: 4.08.2020. 
Table 1. The values of MDA and FRAP in peripheral blood of androgenetic alopecia patients and healthy subjects

\begin{tabular}{lccc}
\hline Parameter & $\begin{array}{c}\text { AGA patients } \\
n=21\end{array}$ & $\begin{array}{c}\text { Healthy subjects } \\
n=30\end{array}$ & $P$-value \\
Me $(\mathbf{Q} 25-\mathbf{Q} 75)$ & Me (Q25-Q75) & \\
\hline MDAe $[\mathrm{nM} / \mathrm{gHb}]$ & $22.22(18.37-29.71)$ & $19.35(14.61-28.73)$ & 0.130 \\
\hline MDA $[\mathrm{nM} / \mathrm{ml}$ plasma $]$ & $0.44(0.42-0.49)$ & $0.42(0.38-0.46)$ & 0.572 \\
\hline FRAP $[\mathrm{mmol} / \mathrm{l}]$ & $212.00(185.00-241.00)$ & $184.00(155.00-224.00)$ & 0.028 \\
\hline
\end{tabular}

AGA - androgenetic alopecia, Me - median, MDA - malondialdehyde concentration in erythrocytes, MDA - malondialdehyde concentration in blood plasma, $p$-statistical significance level (Mann-Whitney U test).

the measurement of oxidative stress and its effects in the body. It provides the necessary information on the reduction power of body fluids [12].

Significant differences in the FRAP levels may result from the body defensive response to the ongoing process of lipid peroxidation. This hypothesis may be supported by a positive correlation between the FRAP level and plasma MDA concentration in patients with AGA.

Taking into account that the relationships between inflammation and oxidative stress are the subject of many studies, the interest in the oxidative-antioxidative balance in the course of AGA seems to be fully understood. This study was aimed at further explanation of the sources of oxidative damage and possible endogenous antioxidative mechanisms occurring in the course of this disease in humans.

\section{Acknowledgments}

The authors thank the Department of Medical Biology and the Department of Biophysics and Medical Physics Division (Nicolaus Copernicus University in Torun) for technical support during the preparation of this manuscript.

\section{Conflict of interest}

The authors declare no conflict of interest.

\section{References}

1. Torres F. Androgenetic, diffuse and senescent alopecia in men: practical evaluation and management. Curr Probl Dermatol 2015; 47: 33-44.

2. Prie BE, Voiculescu VM, Ionescu-Bozdog OB, et al. Oxidative stress and alopecia areata. J Med Life 2015; 8: 43-46.

3. Cranwell W, Sinclair R. Male androgenetic alopecia. Endotext (internet) 2016.

4. Jaworsky C, Kligman AM, Murphy GF. Characterization of inflammatory infiltrates in male pattern alopecia: implications for pathogenesis. Br J Dermatol 1992; 127: 239-46.

5. Whiting DA. Scalp biopsy as a diagnostic and prognostic tool in androgenetic alopecia. Dermatol Ther 1998; 8: 24-33.

6. Sueki H, Stoudemayer T, Kligman AM, et al. Quantitative and ultrastructural analysis of inflammatory infiltrates in male pattern alopecia. Acta Derm Venereol 1999; 79: 347-50.

7. Stefanadi EC, Dimitrakakis G, Antoniou CK, et al. Metabolic syndrome and the skin: a more than superficial association. Reviewing the association between skin diseases and metabolic syndrome and a clinical decision algorithm for high risk patients. Diabetol Metab Syndr 2018; 10: 9.

8. Magro CM, Rossi A, Poe J, et al. The role of inflammation and immunity in the pathogenesis of androgenetic alopecia. J Drugs Dermatol 2011; 10: 1404-11.

9. Aslani FS, Dastgheib L, Banihashemi BM. Hair counts in scalp biopsy of males and females with androgenetic alopecia compared with normal subjects. J Cutan Pathol 2009; 36: 734-9.

10. Bickers DR, Athar M. Oxidative stress in the pathogenesis of skin disease. Int J Dermatol 2006; 126: 2565-75.

11. Pandey KB, Rizvi SI. Biomarkers in oxidative stress in red blood cells. Biomed Pap Med Fac Univ Palacky Olomouc Czech Repub 2011; 155: 131-6.

12. Benzie IFF, Strain JJ. The ferric reducing ability of plasma (FRAP) as a measure of "antioxidant power": the FRAP assay. Anal Biochem 1996; 239: 70-6. 